\title{
Gravidez na Adolescência: Proposta de Intervenção na UBS
}

Pregnancy in Adolescence: Proposal of Intervention in UBS

Embarazo en Adolescente: Propuesta de Intervención en UBS

Avha Clarice Paixão Soares ${ }^{1}$

\section{Resumo}

Objetivo: propor uma intervenção com ações educativas e de busca ativa do público-alvo, com a finalidade de promover uma redução destes índices, através de ações educativas junto aos adolescentes assistidos pela unidade de saúde. Método: Trata-se de um projeto de intervenção, com proposta de intervenção em quatro grandes ações que envolvem a motivação da comunidade através da equipe de saúde para buscar a discussão dos temas. Busca-se integração entre responsáveis e filhos adolescentes, esclarecendo-se dúvidas desse público, além de compartilhar os conceitos de modo correto sobre sexualidade, doenças sexualmente transmissíveis e métodos contraceptivos para esta população, mal informada e carente de diálogo. Resultados: serão obtidos após sua aplicação. Conclusão: As ações desenvolvidas através desse trabalho certamente se tornarão importantes instrumentos para a promoção de saúde e prevenção da gravidez na adolescência e de outros agravos tão impactantes na vida da comunidade.

\section{Palavras-chaves: Estratégia Saúde da Família; Gravidez na Adolescência; Planejamento Familiar.}

\author{
Abstract \\ Objective: To propose an intervention \\ with educational and active target
}

${ }^{1}$ Médica Residente em Ginecologia e Obstetrícia no Hospital Universitário Professor Alberto Antunes da Universidade Federal de Alagoas (HUPAA/UFAL). Autora correspondente: Av. Lourival Melo Mota, s/n Tabuleiro dos Martins, Maceió, AL, Brasil. CEP: 57072-900. E-mail: avhaclarice@ hotmail.com 
audience search, in order to promote a reduction in these rates through educational activities with adolescents assisted by the health unit. Method: This is an intervention project with intervention proposal in four major actions involving the community motivated by health staff to seek discussion of the issues. The aim is to integrate parents/tutors and teens, clarifying doubts whether this public, and share the correct concepts of sexuality, sexually transmitted diseases and contraception in this population, wich is misinformed and lacking in dialogue. Results: will be obtained after application. Conclusion: The actions developed through this work will certainly become important tools for the promotion of health and prevention of teenage pregnancy and other health problems as impactful in the community.

Keywords: Family Health Strategy; Pregnancy in Adolescence; Family Planning.

\section{Resumen}

Objetivo: proponer una intervención con actividades educativas y de búsqueda activa de público adolescente, con el fin de promover una reducción de estos índices, a través de acciones educativas entre los adolescentes con la ayuda de la unidad de salud. Método: este es un proyecto de intervención, con la propuesta de intervención en cuatro acciones principales que implican en la motivación de la comunidad a través del equipo de salud para recoger la discusión de los temas. Promover la integración entre los adolescentes y responsables, clarificar duda además compartir los conceptos correctos sobre sexualidad, enfermedades de transmisión sexual y anticoncepción para esta población, mal informado $y$ con falta de diálogo. Resultados: se obtienen después de su aplicación. Conclusión: Las acciones desarrolladas a través de este trabajo sin duda se convierten en instrumentos importantes para la promoción de la salud y prevención de embarazo adolescente y otros daños impactante en la vida de la comunidad.

Palabras clave: Estrategia de Salud Familiar; Embarazo en Adolescencia; Planificación Familiar.

\section{Introdução}

A adolescência apresenta transformações psicológicas, sexuais, físicas e sociais e esses fatores 
fragilizam os jovens. As influências da mídia e dos grupos sociais, as incertezas e dúvidas, os tabus junto aos pais/responsáveis, dificultam o discernimento de melhores escolhas para o seu futuro ${ }^{(1)}$. A Estratégia de Saúde da Família (ESF) é a porta de entrada do sistema de saúde e inúmeras são as responsabilidades dessa sobre sua população $\operatorname{adscrita}^{(2)}$. Torna-se necessário que as equipes identifiquem principais problemas e proponham ideias de atuação para melhorar a qualidade de vida da população.

A gravidez não planejada, independente da idade, pode trazer dificuldades psicossociais para os pais e, principalmente, para a criança. $\mathrm{O}$ indivíduo fruto da falta de planejamento familiar tem mais chances de sofrer negligência, maus tratos $\mathrm{e}$ até $\mathrm{o}$ abandono. Na adolescência é problema de saúde pública que traz consequências negativas na qualidade de vida das jovens que engravidam ${ }^{(1)}$.

Ao analisarmos a comunidade adscrita da Unidade Básica de Saúde (UBS) Rosete Andrade, percebe-se frequência alta no número de gestantes entre 12 a 19 anos, atingindo o valor de $20 \%$ das gestantes cadastradas e acompanhadas na UBS. Apesar de estar abaixo do valor nacional, que está em média de $26 \%{ }^{(3)}$, é um índice alto e hoje tal situação é tratada como um problema de saúde pública. Observa-se ausência de ações para combater seu aumento dentro da comunidade, e início tardo do pré-natal.

A adolescência do ponto de vista de saúde pública, é marcada por ser um momento de pouca interação entre os serviços de saúde e o público-alvo, muitas vezes devido a dependência dos jovens em seus pais/responsáveis e a moralismos difundidos na sociedade, que impedem a livre procura desses jovens aos serviços ${ }^{(4)}$

Em 1989 foi criado o Programa de Saúde do Adolescente (PROSAD), que tem como intuito a integralidade das ações de forma multiprofissional, intersetorial e interinstitucional, além do foco preventivo e educacional, no atendimento dos jovens/adolescentes, onde uma das áreas de prioridade é a Saúde Reprodutiva e a Sexualidade ${ }^{(4)}$. Esta prioridade ainda não é bem executada, inclusive por profissionais da saúde em seu cotidiano, que não conseguem, junto às suas equipes, lidar com essa população tão sedenta e, ao mesmo tempo, tão carente de informação. Os serviços de saúde ainda não estão devidamente capacitados para encarar os desafios decorrentes da demanda que estes jovens propõem. 
Como consequência da desestruturação da saúde na atenção a esses jovens, somado a fatores sociais importantes como baixo nível socioeconômico, influência da mídia e grupos sociais e outros, a gravidez na adolescência tem atingido valores alarmantes e implica diretamente no futuro dessas jovens, tanto pessoal como profissional $^{(4)}$.

As atividades educativas $\mathrm{e}$ preventivas são essenciais na orientação desses indivíduos quanto a vida sexual e reprodutiva. É importante enfatizar a anticoncepção na adolescência, disponível na atenção básica, não só como proteção contra a gravidez, mas também contra as Doenças Sexualmente Transmissíveis (DST's) e HIV/AIDS ${ }^{(4)}$. A gestação em adolescentes, relacionase com alto índice de evasão escolar e a literatura aponta que apenas $53 \%$ das adolescentes que engravidam conseguem terminar o segundo grau, em comparação aos $95 \%$ daquelas que não engravidam $^{(5)}$.

Na comunidade adscrita a UBS estudada a taxa de nascidos vivos entre mães entre 10 e 19 anos é de $20 \%$. Mesmo abaixo do nível nacional, o valor é considerado alto para padrões internacionais. Implicações como abandono da escola, maior dificuldade de inserção no mercado de trabalho, diminuição do padrão de vida, desestruturação familiar e consequente circularidade da pobreza são ocorrentes na gravidez precoce ${ }^{(6)}$.

Puérperas apresentam poucos conhecimentos sobre DST/AIDS e os modos de prevenção ainda são insipientes. Além da deficiência na divulgação de informações e orientação sobre as opções de métodos contraceptivos existentes, aliados à prevenção da gravidez na adolescência $^{(6)}$.

Após entrevistas com os participantes e análise de dados recolhidos na unidade, viu-se a possibilidade de aplicar-se este projeto, além de verificar a veracidade da influência dos nós críticos identificados e descritos como causadores da gravidez na adolescência.

\section{Diagnóstico Situacional}

Penedo é uma cidade antiga às margens do Rio São Francisco, e é cidade histórica do Brasil. Situada a 157 km de Maceió, é intitulada "Ouro Preto do Nordeste" graças a seu riquíssimo acervo arquitetônico presente em sobrados e igrejas seculares.

O Índice de Desenvolvimento Humano (IDH) registrado em 2007 foi de 0,665, sendo o sexto em Alagoas, 
apresentando um rendimento familiar médio de $\mathrm{R} \$ 1462,86$ na área urbana e $\mathrm{R} \$ 650,12$ na área rural. Penedo possui uma área total de $689,160 \mathrm{~km}^{2}$, com uma população de 60.378 habitantes, distribuídas em 16.333 famílias, sendo a maioria da população residente na área urbana $(74,6 \%)$ e $25,4 \%$ na área rural ${ }^{(7)}$.

As principais atividades econômicas provêm da atividade primária, como coco, o arroz, a pesca e a cana-de-açúcar, e uma parcela da fonte de renda advém do turismo local. A evasão da área rural é uma realidade brasileira, e Penedo não foge do padrão, contribuindo para o crescimento desordenado das cidades. A falta de investimento na agricultura familiar contribui para esta migração.

A cidade possui uma densidade demográfica de 87,61 hab./ $\mathrm{km}^{2}$, taxa de alfabetização de 70,2\%. Conta com 16.355 domicílios particulares, dos quais $92,29 \%$ possui banheiro ou sanitário e destes, apenas $11,64 \%$ possui banheiro e esgotamento sanitária via rede geral. Cerca de 93,28\% são abastecidos pela rede geral de água, enquanto que $3,76 \%$ são abastecidos por poço ou nascente e $2,98 \%$ utiliza outras formas de abastecimento. Apenas $86,70 \%$ dos domicílios são tem coleta de lixo, evidenciando fonte de problemas ambientais e de saúde pública.

A UBS Rosete Andrade é responsável por uma área, dividida em 8 microáreas. Equipada com computador e um funcionário exclusivo para marcação de consultas especializadas disponíveis no município, algumas concentradas no ambulatório municipal - Centro de Especialidades de Penedo (CESPE) - e outras em instituições conveniadas, e de exames complementares através do sistema tecnológico unificado, o que diminui o deslocamento deste paciente. A marcação de alguns exames e especialistas que não constam no município, ainda estão centralizados na Secretaria Municipal de Saúde, onde o paciente deve ele mesmo ir para providenciar a marcação.

Conta com apoio do Núcleo de Apoio a Saúde da Família (NASF), que fica a cerca de $2 \mathrm{~km}$ de distância do posto, além de um Centro de Assistência Psicossocial (CAPS), porém ainda tem dificuldade em manter integração entre os setores. Isto ocorre devido ao não recebimento da contrarreferência, o que leva a recorrer às informações, por vezes incompletas, dos próprios pacientes, problema que poderia ser solucionado com um 
sistema computadorizado e integrado de prontuários multi-disciplinares.

Para a realização das visitas domiciliares dispõe de uma viatura exclusiva para a unidade uma vez por semana. A estrutura física do posto é adequada, principalmente por ter sido recém-reformado, possuindo salas climatizadas, em boas condições e exclusivas para as diferentes funções (sala de vacina, sala de curativo, sala de consulta médica, sala de consulta com enfermeira, farmácia, sala de arquivo, almoxarifado, banheiros em ótimas condições de uso, sala de espera ampla e com assentos disponíveis para a dimensão do fluxo do posto).

$\mathrm{Na}$ área de abrangência da UBS existem 5780 habitantes, divididos em 1120 famílias. O nível de alfabetização é $69,4 \%{ }^{(6)}$, demonstrando uma carência educacional na região, principalmente na população adulta. A comunidade conta com duas escolas públicas municipais e uma estadual, além de 3 igrejas católicas e 2 evangélicas e serviços básicos como luz elétrica, água e telefonia, não constando com estabelecimentos bancários e correios.

O índice de natalidade da população da UBS está em níveis elevados, principalmente entre as adolescentes, fato percebido durante o dia-a-dia da equipe de saúde da família. Encontrou-se significativo número de gestantes adolescentes

identificadas na área adscrita em 2013, através de dados obtidos por meio de consulta ao Sistema de Acompanhamento do Programa de Humanização no Pré-natal (SISPRENATAL). Usou-se também os registros da equipe da UBS (prontuários, fichas de atendimento, relatórios e outros) para elaboração do Diagnóstico Situacional da Unidade.

Um agravante é que, destas 5 gestantes abaixo dos 20 anos, 3 delas estão na segunda gestação, e das outras 20 gestantes, hoje com mais de 20 anos, 7 estão na segunda gestação e tiveram a primeira com menos de 20 anos. A literatura comprova que adolescentes que engravidam apresentam mais riscos a sua saúde com maior incidência de anemia materna, doença hipertensiva específica da gravidez, desproporção céfalo-pélvica, infecção urinária, prematuridade, placenta prévia, baixo peso ao nascer, sofrimento fetal agudo intraparto, complicações no parto e puerpério $^{(4)}$.

Em relação a educação formal, é visto que o percentual de adolescentes que engravidam e conseguem terminar o segundo grau é bem menor comparando-se com aquelas que não 
engravidam neste período de vida ${ }^{(5)}$. O que traz consequências pessoais e econômicas, perpetuando um estado de baixo nível socioeconômico entre essa população. Este é mais de um dos motivos que mostra a necessidade de políticas públicas eficientes para evitar o acréscimo desta situação

Segundo dados do DATASUS $^{(3)}$, no Brasil $26 \%$ dos nascidos vivos são filhos de garotas entre 10 a 19 anos. Em Alagoas o índice é de $27,4 \%$, contribuindo para a evasão escolar de meninas grávidas e aumento da pobreza. Revendo os dados apenas da cidade de Penedo, das 1242 mulheres grávidas, $24,79 \%$ eram adolescentes. Apesar de a incidência de gestantes adolescentes da área de abrangência desta USF ser abaixo da taxa nacional, este problema social causa impacto direto em diversos setores da vida pessoal e profissional destas jovens e suas famílias.

Situação Problema

Descobriu-se diversos nós críticos, que são responsáveis pelo alto índice de gestação na adolescência na área de abrangência da UBS como: baixo nível socioeconômico, ausência/ desconhecimento do planejamento familiar, cultura intrínseca da população de que "menina tem que ter filho e casar", falta de educação sexual desde o ensino escolar, influência da mídia e grupos de amigos para início da atividade sexual, sentimentos de vergonha e medo perante os familiares por ter iniciado a vida sexual, causando receio de procurar assistência profissional para sanar dúvidas ou iniciar métodos anticonceptivos,

Estes nós críticos foram identificados depois de várias discussões entre os membros da equipe de saúde em questão, o que justifica este trabalho. Como prioridade, escolheu-se este problema para propor projetos $\mathrm{e}$ ações com o intuito de reduzir este indicador, pois observa-se ausência de ações para combater seu aumento dentro da comunidade, comparado aos outros problemas encontrados que já dispõem de ações resolutivas em prática, além de ter sido constatado a alta prevalência de adolescentes grávidas e que iniciavam o pré-natal tardiamente, muitas vezes, pelo medo de assumir esta condição junto aos seus familiares ou pelo desconhecimento do próprio corpo.

\section{Objetivos}

\section{Geral}


Propor um plano de intervenção para reduzir os elevados índices de gravidez na adolescência.

\section{Específicos}

Analisar a literatura relacionada a gravidez na adolescência.

Propor estratégias para reduzir os índices de gravidez na adolescência, DST's, infecção pelo HIV.

Propor estratégias para conscientizar os adolescentes dos riscos e consequências da gravidez nesta faixa etária.

\section{Método}

O plano de ação foi elaborado pela autora com o auxílio da equipe de saúde na qual a autora está inserida. Antes de pôr em prática o plano será necessário capacitar os profissionais juntamente com a equipe de psicólogos do NASF para orientar toda a equipe sobre os temas abordados e sobre a melhor atitude para ter diante dos convidados.

\section{Proposta de projeto de intervenção}

O Projeto "Pais Amigos" visa aumentar a comunicação entre pais e filhos adolescentes. Através do intermédio da equipe de saúde, observar-se-á as atitudes dos participantes na discussão de temas sexo e sexualidade, e intervir de modo a melhorar essa comunicação. Pretende-se realizar reuniões semanais, durante 3 semanas do mês, agendadas de acordo com o cronograma da equipe de saúde, com grupos de 30 adolescentes, entre 15 e 19 anos, e seus respectivos pais e/ou responsáveis, escolhidos aleatoriamente e convidados pelos ACS. As reuniões terão a duração média de uma hora.

A coordenação do encontro será feita pela médica da equipe, com participação dos demais membros da equipe. Pretende-se realizar 4 encontros com cada grupo de adolescentes, ocorrendo o revezamento dos grupos montados de acordo com a quantidade de adolescentes da área adscrita; atuando em vários grupos ao mesmo tempo. O conhecimento e práticas de educação em saúde adquiridos durante a atividade cotidiana dos profissionais, juntamente a capacitação prévia, serão fundamentais para acrescer as discussões dos encontros. No primeiro encontro, será elaborado um cronograma com temas como: "Saúde Reprodutiva", "Sexo e Sexualidade", "Métodos Contraceptivos e DST's", "Importância do Diálogo", etc. Ao final dos encontros, uma reflexão com os participantes para avaliar a qualidade. 
A ação denominada "Tempo Certo, Vida Certa" objetiva a criação de grupos de discussão para que os adolescentes tenham um espaço aberto para debater diretamente temas como a atividade sexual precoce, a influência dos amigos e da mídia e as consequências da gestação precoce devido a atitudes impensadas. Através da montagem dos grupos do projeto "Pais Amigos", capta-se jovens para participar neste outro tipo de encontro, com maior liberdade para que expressem suas ideias, incertezas e dúvidas. Aqui as reuniões serão mensais, totalizando 6 encontros com espaço aberto para os desejem frequentá-las, contando a motivação do convite pelo ACS.

O projeto "Cuidar para Viver" objetiva elevar o nível de informação dos adolescentes sobre a própria saúde reprodutiva, doença sexualmente transmissíveis, métodos anticoncepcionais. Capacitar profissionais da equipe para utilizar os recursos pedagógicos a serem utilizados (folhetos, cartazes, palestras) é importante para a adequar a transmissão de conhecimentos. Essas reuniões também serão mensais, (seis encontros). Ao final, será feita a avaliação do entendimento do grupo sobre os temas e sobre a qualidade dos encontros.
Por fim, o projeto "Entendendo o Corpo e a Sexualidade" pretende transmitir conhecimentos para $\mathrm{O}$ público-alvo em um nicho favorável às primeiras descobertas sexuais: a escola. A educação sexual na escola vem se mostrando uma grande ferramenta de esclarecimento dos jovens, porém, a ocorrência desses eventos depende de articulação com a diretoria das escolas, com o conselho escolar e com a opinião dos pais sobre a abordagem. Pretende-se realizar três eventos, um em cada escola da comunidade, outra maneira de divulgação da existência dos demais projetos.

\section{Resultados}

Após a implementação da proposta de intervenção, serão realizadas avaliações semestrais do plano de ação. A avaliação será pautada no número de gestantes adolescentes grávidas captadas e tempo decorrido de captação pelos ACS em cada microárea.

Outro ponto de avaliação do plano será revisão, trimestral, das gestantes adolescentes captadas precocemente (no primeiro trimestre de gravidez), dos adolescentes identificados e classificados em situação de risco e vulnerabilidades sociais na área 
abrangência da UBS, onde se espera o alcance da meta proposta que é a redução da incidência de gravidez nesta faixa etária.

Espera-se os resultados abaixo descritos: Pais Amigos: sensibilização dos responsáveis quanto a importância da comunicação no seio familiar; promoção da troca de experiências entre os familiares; Tempo Certo, Vida Certa: atingir $100 \%$ dos adolescentes da área adscrita com mais e melhor conhecimento e controle sobre a sua sexualidade, gravidez e DST's; Cuidar para Viver: adolescentes mais preparados para tomada de decisões conscientes; Entendendo o Corpo e a Sexualidade: parceria entre o setor educação e saúde e promoção a cidadania.

\section{Conclusão}

As ações desenvolvidas através desse trabalho se tornarão importantes instrumentos para a promoção de saúde e prevenção da gravidez na adolescência e de outros agravos tão impactantes na vida da comunidade.
Adolescentes estudantes: conhecimentos das complicações do aborto provocado. Rev. Gaúcha de Enferm. 465-71.

2011;32(3):

2. Brasil. Ministério da Saúde. Secretaria de Atenção à Saúde. Departamento de Atenção Básica. Política Nacional de Atenção Básica. Brasília: Ministério da Saúde, Série E: Legislação da Saúde. 2012. 110 p.

3. Ministério da Saúde. Datasus.2011. Disponível em: http://www.datasus.gov. br/>. Acesso em: 10 de dezembro 2013.

4. Brasil, Ministério da Saúde: Secretaria de Atenção à Saúde, Departamento de Atenção Básica, Cadernos de Atenção Básica $\mathrm{N}^{\circ}$ 26: Saúde Sexual e Saúde Reprodutiva. Brasília; 2010.

5. Yazlle, M. E. H. D. Gravidez na adolescência. Rev. Bras. Ginecol. Obstet. 2006; 28(8):443-5.

6. Andrade, M. L. R; Brito M.C.C. Freitas C.A.S.L. Planejamento familiar: um recurso estratégico à maternidade responsável de adolescentes primíparas. SANARE - Revista de Políticas Públicas. 2013; 12(1):27-32.

7. IBGE. Censo Demográfico de 2010. Disponível em: <http://censo2010.ibge. gov.br>. Acesso em: 2 de fevereiro. 2013.

\section{Referências}

1. Correia D.S., Monteiro V.G.N., Cavalcante J.C., Maia E.M. C. 